\title{
Research on Testability Growth Modeling Method Based on Gompertz Function
}

\author{
Yixin $\mathrm{Hu}^{1}$, Zhenpeng $\mathrm{He}^{2}$, Baichun $\mathrm{Li}^{2}$, Yong $\mathrm{Liu}^{2}$, Junze Qian ${ }^{1}$ \\ ${ }^{1}$ Engineering Techniques Training Center, Civil Aviation University of China, Tianjin 300300, China \\ ${ }^{2}$ College of Aeronautical Engineering, Civil Aviation University of China, Tianjin 300300, China \\ *Corresponding Author: Yixin Hu (E-mail: yxhu@cauc.edu.cn)
}

\begin{abstract}
Improving the testability level of equipment is of great significance for improving and perfecting the work of testability design. In order to improve the testability level and ensure the effective growth of equipment testability, a testability growth model of equipment based on Gompertz function is proposed, and the Virene algorithm is used to estimate the key parameters in the model, thereby establishing an effective and feasible predictive model. By solving the proposed model, the purpose of predicting the future testability level of equipment is achieved. Experiments show that the proposed model can reasonably predict the future testability level, which provides a strong theoretical basis for the testability design improvement of equipment.
\end{abstract}

Keywords: Testability, Testability Design, Testability Growth Model, Gompertz.

\section{Introduction}

Testability is a design feature that a product can determine its status (workable, non-workable, or performance degradation) in a timely and accurate manner, and isolate its internal faults. Similar to the characteristics of reliability, maintainability, supportability and safety, testability is one of the general quality characteristics of equipment[1-4]. Testability Growth (TG) is an important part of testability design, and an effective way to improve product testability and enhance its testability indicators. Testability Growth Model (TGM) is an analytic, parametric, and time variable function. A good TGM can track and predict the testability level well. Based on the TGM, the testability designer can plan TG test and make some decisions like the time to stop testing or continue to test[5]. Testability level is usually measured by testability indicators, which usually include Fault Detection Rate (FDR) and Fault Isolation Rate (FIR).

With the increasing requirements of equipment systems for operational reliability, safety, life cycle costs (LCC), etc., it is essential to establish a reasonable and effective TGM based on the current testability level, so as to effectively predict whether the equipment can continue to meet the testability requirements in the future. How to establish a reasonable and effective TGM has gradually become a research hotspot for scholars at home and abroad. Li et al. ignored the correction delay process of testability design defects for the existing TGM, which led to TGM tracking and to predict the problem of low accuracy, a TGM modeling theory and method that considers the delay of testability design defect correction was proposed[6]; Wang et al. proposed a compatibility method based on Fisher statistics for TG test data, fisher statistics is constructed according to the hypergeometric distribution to judge the joint relationship between adjacent growth stages, and then the relevant conclusions on the overall consistency between growth test data and field data are obtained[7]; Jiang established a testability analysis and verification process system and implementation steps, and applied it in TG process of product, the effective growth of testability was guaranteed[8]. Literature[9] provides an interval of existence of negative critical mortality rate parameters in Gompertz survival model, in the absence of age specific mortality data. Yan proposed a prediction model based on Gompertz function and customer life cycle, finally, the number of outer-net mobile customers is predicted accurately and dynamically[10].

The above proposed different model building methods for TG, however, no one has applied Gompertz model to the TG process of equipment. TGM of equipment has always been one of the considerations, which is in line with the characteristics of the S-shaped growth curve, and the Gompertz model is a relatively common model to describe TG. Therefore, this paper proposes the Gompertz model of TG and verifies the validity and rationality of the proposed model through test routines.

\section{TGM Construction Method Based on Gompertz Function}

Gompertz function was first proposed by the British statistician Benjamin Gompertz and used as a growth model of animal populations to describe the extinction law of populations[11]. The model is characterized by slow growth in the initial curve and a steady upward trend, and rapid growth in the mid-term, and a rapid upward trend. The growth area tends to be saturated after the inflection point in the later period, the curve develops in a horizontal trend, and the overall curve is an S-shaped curve, as shown in Figure 1.

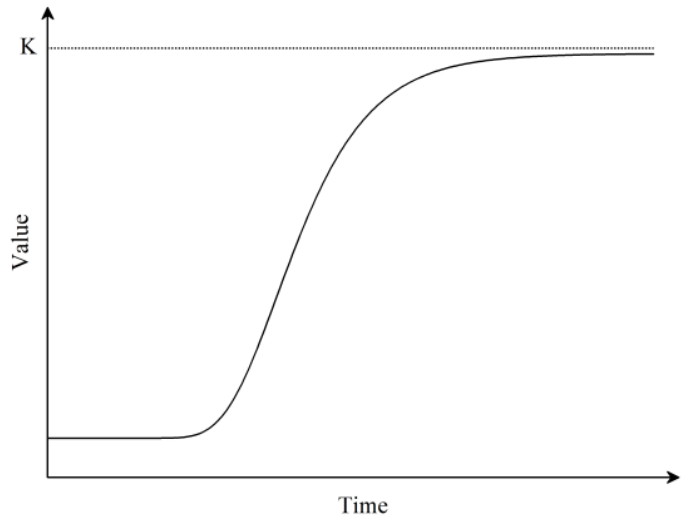

Figure 1: Gompertz curve model diagram 
In 1968, E. P. Virene used the Gompertz model curve to describe the increasing law of reliability. Nowadays Gompertz model is widely used in economics, demography, population ecology, medicine, physiology, genetics, chemistry, forestry and many other fields. Gompertz model can initially assess the current testability level of equipment and can predict the future testability level. It's a discrete growth model. Combining with the characteristics of TG, assuming that the testability level in time (or times or stages) is $P(t)$ (FDR or FIR value), the basic formula for establishing a TG model based on Gompertz is as Equation (1):

$$
P(t)=K a^{b^{t}}
$$

where the parameter $K$ represents the extreme value of TG, when $t \rightarrow \infty, P(t)=K$ is established; and when $t \rightarrow 0$, $P(0)=K a$ is established, then $K a$ represents the initial testability level of equipment; the parameter $a$ is the ratio of the initial testability level to the upper limit of $\mathrm{TG}$; the parameter $b$ represents the speed of TG, the smaller the value $b$, the faster the growth rate, and vice versa. Besides, the value ranges of the parameters $K, \quad a$ and $b$ are all $(0,1)$.

\section{Method of Estimating TGM Parameters}

E. P. Virene first proposed the Virene algorithm to estimate the critical parameters in Gompertz model[12]. Assuming that there are known $n=3 m$ observations $P_{i}(i=0,1,2 \ldots, 3 m-1)$, if $n$ is not divisible by three, individual data can be increased or decreased to make it a multiple of three, and the values of $K, a, b$ in the model need to be solved. Since the formula $P_{i}=K a^{b^{i}}$ is established, taking the logarithm of both sides at the same time, we can get Equation (2):

$$
\ln P_{i}=\ln K+b^{i} \ln a \quad(i=0,1,2 \ldots, 3 m-1)
$$

the $n$ observations are divided into three groups, each group has $m$ values, then the following formulas can be obtained:

$$
\begin{aligned}
& Q_{1}=\sum_{i=0}^{m-1} \ln P_{i}=m \ln K+\ln a \sum_{i=0}^{m-1} b^{i} \\
& Q_{2}=\sum_{i=m}^{2 m-1} \ln P_{i}=m \ln K+\ln a \sum_{i=m}^{2 m-1} b^{i} \\
& Q_{3}=\sum_{i=2 m}^{3 m-1} \ln P_{i}=m \ln K+\ln a \sum_{i=2 m}^{3 m-1} b^{i}
\end{aligned}
$$

Equation (6) can be calculated by formulas (3), (4), (5), as shown below:

$$
\frac{Q_{3}-Q_{2}}{Q_{2}-Q_{1}}=\frac{\sum_{i=2 m}^{3 m-1} b^{i}-\sum_{i=m}^{2 m-1} b^{i}}{\sum_{i=m}^{2 m-1} b^{i}-\sum_{i=0}^{m-1} b^{i}}=b^{m}
$$

the estimated value of parameter $b$ can be obtained by solving Equation (6) as shown in Equation (7).

$$
\hat{b}=\left[\frac{Q_{3}-Q_{2}}{Q_{2}-Q_{1}}\right]^{\frac{1}{m}}
$$

The estimated value of parameter $K$ and $a$ can be calculated continuously, by

$$
\begin{aligned}
& Q_{1}-m \ln K=\ln a \sum_{i=0}^{m-1} b^{i} \\
& Q_{2}-m \ln K=\ln a \sum_{i=m}^{2 m-1} b^{i}
\end{aligned}
$$

We can get Equations (10) - (12) as shown below:

$$
\begin{gathered}
\frac{Q_{2}-m \ln K}{Q_{1}-m \ln K}=b^{m} \\
\ln K=\frac{1}{m}\left[Q_{1}+\frac{Q_{2}-Q_{1}}{1-b^{m}}\right] \\
\hat{K}=\exp \left\{\frac{1}{m}\left[Q_{1}+\frac{Q_{2}-Q_{1}}{1-b^{m}}\right]\right\}
\end{gathered}
$$

Because,

$$
\begin{aligned}
& Q_{1}-\ln a \sum_{i=0}^{m-1} b^{i}=m \ln K \\
& Q_{2}-\ln a \sum_{i=m}^{2 m-1} b^{i}=m \ln K
\end{aligned}
$$

The estimated value of parameter $a$ can be obtained by calculation as shown in Equation (17):

$$
\begin{gathered}
Q_{1}-\ln a \sum_{i=0}^{m-1} b^{i}=Q_{2}-\ln a \sum_{i=m}^{2 m-1} b^{i} \\
\ln a=\frac{\left(S_{2}-S_{1}\right)(b-1)}{\left(1-b^{m}\right)^{2}} \\
\hat{a}=\exp \left\{\frac{\left(S_{2}-S_{1}\right)(b-1)}{\left(1-b^{m}\right)^{2}}\right\}
\end{gathered}
$$

By using Virene algorithm, the estimated values of parameters $K, \quad a$ and $b$ in TGM model are $\hat{K}, \hat{a}$ and $\hat{b}$, respectively, and the TGM model formula based on Gompertz can be rewritten as:

$$
\hat{P}(t)=\hat{K} \hat{a}^{\hat{b}^{t}}
$$

\section{Applications}

In the engineering application of testability design, FDR and FIR are commonly used to measure the TG level. It's assumed that the TG test cycle of a certain equipment is twelve stages. The FDR and FIR data of the first six stages are currently known as shown in Table 1. Corresponding improvements are required after each test stage in order to gradually improve the testability. After the end of the test, the FDR and FIR index levels are not less than 0.95 and 0.98 respectively. For this purpose, the TGM model is solved determine how well the known testability meets the actual testability, and predict whether the future testability level meets the requirements in 
the final stage.

It is necessary to first determine the three parameters in TGM. For this reason, the six-stage FDR and FIR data are divided into three groups. The two data numbers in each group are shown in Table 1. Calculated by Equations (3)-(5), the following calculation results can be obtained:

$$
\begin{array}{r}
\left\{\begin{array}{l}
Q_{F D R, 1}=\ln \left(P_{F D R, 0} * P_{F D R, 1}\right)=-0.9161 \\
Q_{F D R, 2}=\ln \left(P_{F D R, 2} * P_{F D R, 3}\right)=-0.1621 \\
Q_{F D R, 3}=\ln \left(P_{F D R, 4} * P_{F D R, 5}\right)=-0.0537
\end{array}\right. \\
\left\{\begin{array}{l}
Q_{F I R, 1}=\ln \left(P_{F I R, 0} * P_{F I R, 1}\right)=-0.6036 \\
Q_{F I R, 2}=\ln \left(P_{F I R, 2} * P_{F I R, 3}\right)=-0.0657 \\
Q_{F I R, 3}=\ln \left(P_{F I R, 4} * P_{F I R, 5}\right)=-0.0208
\end{array}\right.
\end{array}
$$

According to equations (7), (12) and (17), we can obtain:

$$
\left\{\begin{array}{l}
\hat{K}_{F D R}=0.9824 \\
\hat{a}_{F D R}=0.5281 \\
\hat{b}_{F D R}=0.3793
\end{array}\right.
$$

$$
\left\{\begin{array}{l}
\hat{K}_{F I R}=0.9917 \\
\hat{a}_{F I R}=0.6342 \\
\hat{b}_{F I R}=0.2889
\end{array}\right.
$$

Substituting the above parameters into Equation (18) to obtain the TGM of FDR and FIR are as follows:

$$
\begin{aligned}
& \hat{P}_{F D R}(t)=0.9824 * 0.5281^{0.3793^{t}} \\
& \hat{P}_{F I R}(t)=0.9917 * 0.6324^{0.2889^{t}}
\end{aligned}
$$

According to Equations (23) and (24), substituting the data, the predicted FDR and FIR values of the equipment in the twelve test phases are $\hat{P}_{F D R, i}$ and $\hat{P}_{F I R, i}$ respectively. Besides,

\begin{tabular}{|c|c|c|c|c|c|c|c|}
\hline Group Number & Test stage & $P_{F D R, i}$ & $\hat{P}_{F D R, i}$ & $\hat{P}_{F D R, i}-P_{F D R, i}$ & $P_{F I R, i}$ & $\hat{P}_{F I R, i}$ & $\hat{P}_{F I R, i}-P_{F I R, i}$ \\
\hline 0 & 0 & 0.4833 & 0.5188 & 0.0355 & 0.5938 & 0.6290 & 0.0352 \\
\hline 0 & 1 & 0.8278 & 0.7712 & -0.0566 & 0.9209 & 0.8695 & -0.0515 \\
\hline 1 & 2 & 0.8967 & 0.8962 & -0.0004 & 0.9562 & 0.9547 & -0.0015 \\
\hline 1 & 3 & 0.9483 & 0.9488 & 0.0005 & 0.9793 & 0.9808 & 0.0015 \\
\hline 2 & 4 & 0.9656 & 0.9695 & 0.0040 & 0.9864 & 0.9885 & 0.0021 \\
\hline \multirow[t]{7}{*}{2} & 5 & 0.9815 & 0.9775 & -0.0040 & 0.9929 & 0.9908 & -0.0021 \\
\hline & 6 & & 0.9806 & & & 0.9914 & \\
\hline & 7 & & 0.9817 & & & 0.9916 & \\
\hline & 8 & & 0.9822 & & & 0.9916 & \\
\hline & 9 & & 0.9823 & & & 0.9917 & \\
\hline & 10 & & 0.9824 & & & 0.9917 & \\
\hline & 11 & & 0.9824 & & & 0.9917 & \\
\hline
\end{tabular}
the difference between the predicted value and the actual value of the first six test stages is $\hat{P}_{F D R, i}-P_{F D R, i}$ and $\hat{P}_{F I R, i}-P_{F I R, i}$ respectively, as shown in Table 1 , and the fitting comparison charts are shown in Figure 2.

Table 1: Test data of testability growth (TG)

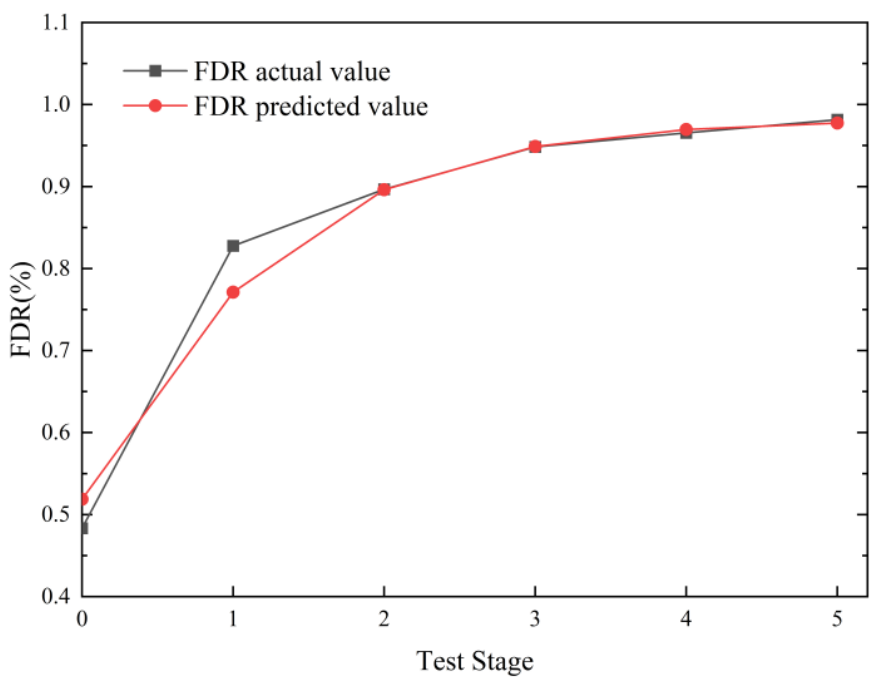

(a)

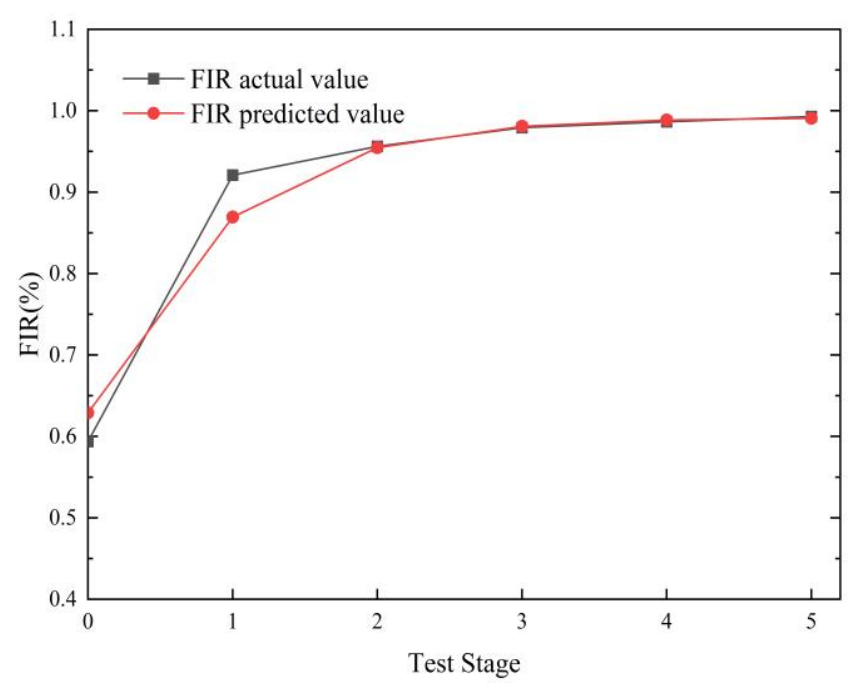

(b)

Figure 2: Comparison of FDR and FIR actual value and predicted value fit

From the data results in Table 1 , it can be seen that the testability level calculated by using the Gompertz-based TGM model proposed meets the requirements, and the final FDR value is greater than 0.95 , and the FIR value is greater than 0.98 , which meets the index requirements. Additionally, from the comparison of difference between actual value and predicted value of FDR and FIR in the first six stages, it is found that the absolute value of the error is less than 0.06. In addition, it can be seen from the comparison chart in Figure 1 that the actual value of FDR and FIR fits the predicted value better. It shows that it is reasonable and effective to use the proposed TGM for TG prediction. 


\section{Conclusion}

TG is an important content of testability design. In order to predict the testability level of equipment in the future, a TG model based on Gompertz model is proposed in this paper. Firstly, a TGM model for TG process is established, and then the Virene algorithm is used to perform numerical calculations on the model, and the key parameters in the TGM are estimated. Finally, an application example verifies the validity and rationality of the proposed TG model. The results show that the proposed TGM can reasonably predict the testability level of equipment in the future stage, and can be used as an important reference for practical engineering applications.

Excellent TGM solving algorithms can achieve a better testability level of prediction accuracy. Therefore, this paper will be extended from the following aspect in the future research: We will find more excellent TGM solving algorithms, and select the best model solving algorithm by comparison. In addition, more experimental data will be collected to verify the proposed TGM.

\section{Acknowledgments}

This work was financially supported by the Fundamental Research Funds for the Central Universities, Special Project of Civil Aviation University of China under Grant 3122019109.

\section{References}

[1] "Testability Program for Electronic Systems and Equipment," Military Standard MIL-STD-2165, 1985.

[2] "General Requirement for Material Testability Program," GJB2547A-2012, 2012.

[3] J.-Y. Shi, Z. Tian, and W.-K. Hou, "Testability design analysis and verification," National Defense Industry Press, Beijing, 2011.

[4] H.-Y. Xie, J. Qiu, and P. Yang, "Testability index allocation method considering unit mutual test," System Engineering and Electronics, vol. 41, no. 12, pp. 2899-2904, 2019.

[5] T. Li, X. Si, Z. Yang, H. Pei and Y. Ma, "NHPP testability growth model considering testability growth effort, rectifying delay, and imperfect correction," IEEE Access, vol. 8, pp. 9072-9083, 2020.

[6] T.-M. Li, X.-S. Si, Z.-H. Yang, C.-Q. Xu, and Q. Zhang. "Foundation and assessment of testability growth model considering testability design limitation rectifying delay function," Acta Aeronautica et Astronautica Sinica, vol, 40, no. 9, pp. 308-316, 2019.

[7] J. Wang, H.-F. He, T.-M. Li, C.-Q. Xu, Z.-H. Yang, C.-C. Han. "The method of compatibility check of testability growth test data based on Fisher-statistics," CHINA MEASUREMENT \& TEST, vol. 43, No. 8, pp. 11-13+40, 2017.

[8] M. Jiang. "Application of Testability Analysis and Verification in Testability Growth," The 17th China Aviation measurement and Control Technology Annual Conference, pp. 451-455, 2020.
[9] E.-S. Lakshminarayanan, M. Pitchaimani. "Existence and estimation of critical negative Gompertz parameter," Computers \& Mathematics with Applications, vol. 55, no. 6, pp. 1686-92, 2008.

[10]Z. Yan, "Prediction model based on Gompertz function," 2009 2nd IEEE International Conference on Broadband Network \& Multimedia Technology, pp. 898-893, 2009.

[11]B. Gompertz, "On the nature of the function expressive of the law of human mortality and on a new mode of determining the value of life contingencies," Philos. Trans. R. Soc, vol. 115, pp. 513-585, 1825.

[12] Y.-Q. Zhou, C.-X. Weng. "Reliability Growth", Beijing Science Press, 1992. 